\title{
Multipath Bandwidth Capacity Allocation and MPLS Internet Traffic Engineering
}

\author{
Hatim S. Hussein
}

\begin{abstract}
The In this paper a description of a new algorithm is introduced to help in solving bandwidth distribution across multipath MPLS network. The intension is to reduce networks operational costs, optimize networks links utilization and attain reliable and fast user Internet connection. Traffic engineering (TE) has proven to be a vital instrument that orchestrated many improvement to network performance in general and achieved countless implementation of Internet applications. TE has become one of the most important tools that help in shaping Internet backbone design and traffic optimization. The objective of this research is to achieve efficient and optimized bandwidth allocation and load sharing across multipath MPLS network. Such solution is an attempt to minimize network congestion, maximize links capacity utilization and improve network
\end{abstract}

Index Terms-Network, protocols, bandwidth allocation, traffic engineering.

\section{INTRODUCTION}

In the last few years, the Internet has become an economic engine, business platform, global entertainment center. The business growth has expanded exponentially [1]. For instance, Internet usage in the United States grow by more than $150 \%$ between the years of 2000 and 2012.. This growth was observed in both business setting and at telecom user's level. In both cases a faster Internet connection was desirable which implies more bandwidth is needed. For instance almost $80 \%$ of the total population is Internet users. On the other hand, compared to the rest of the world growth which was $32 \%$ during the same time frame. As a result of these dramatic changes, it is no surprise that the Internet has become one of the most studied and researched fields in the last decade. Research in areas such as hardware, wireless and applications software. End users are demanding reliable connectivity, and fast throughput, and trusted data transmission from their telecommunication and service providers. In addition to traditional Internet applications such as web browsing, file transfer, and electronic mail, new multicast, multimedia, entertainments, and voice service applications are in the rise. Such applications are bandwidth intensive, connection-oriented at the transport layer, and the majority of them are real time applications. Along with the exponential growth of the network, these new applications put even more strain on the current network resources. The Internet Protocol (IP) [2] has been unable to cope with the changes and adapt to dynamic nature of the Internet and its applications. Traffic Engineering (IE) became an imminent need to optimize and

Manuscript received February 23, 2015; revised August 4, 2015.

Hatim S. Hussein is with George Mason University, US (e-mail: hatimsh@gmail.com). improve the Internet performance.

Network traffic delays and the limited bandwidth availability are two of the main issues the Internet faces today. Internet traffic engineering and adaptable bandwidth allocation can be seen as an attempt to resolves these type of problems. This paper tries to describe and shed some light on this problem, with an attempt to propose a traffic engineering solution using multipath bandwidth allocation algorithm. Such algorithm will be based on link capacity, reserved bandwidth, and bandwidth availably, and other network configurable parameters.

\section{RELATED WORK}

\section{A. Interior Gateway Protocols (IGP)}

Network traffic is forwarded by Interior Gateway Protocol (IGP) based on the available path chosen. Fig. 1, shows that when traffic is transmitted from S1 to node D1, traffic is traversed through $\mathrm{M} 2$ since it has a lower cost of 30 compared to 40 , via node M3. Not only the payload heading to devices connected to network D1 take the shortest path, but also any network traffic sent to hosts connected to router D2 and D3.

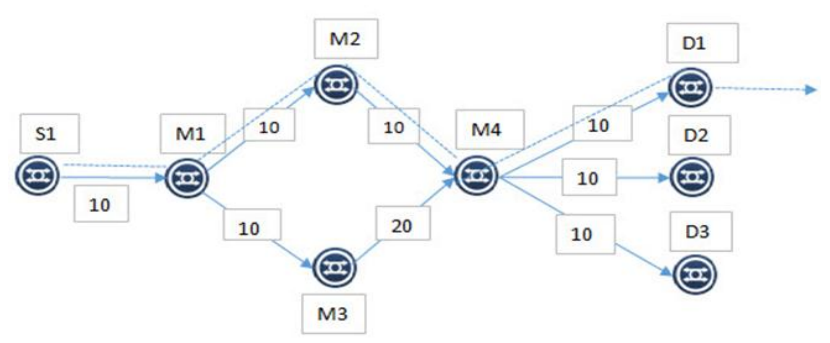

Fig. 1. IGP routing process.

Transmitting all network traffic for networks D1, D2, and D3 via node M2 may make M2 the bottleneck and render the path through M3 underused. For whatever reason you may want to send of the network payload via M3. The only way to send network traffic to router D3 via M3 is to increase the cost for the link between M2 and M4 to say 21, as show in Fig. 2. By increasing the cost and forcing the traffic to traverse the lower path may leads to undesired results.

IGP routing techniques are cost-based or hop counts routing protocols, they don't take into account the condition of the network such as delays, congestions, or traffic on the network. Due to the nature of these protocols, network traffic will not be allocated evenly across multiple paths throughout the network. Some paths may become over utilized and overs may be underutilized. With today's Internet the need for traffic engineering to control the paths network traffic traverse so load sharing and load balance can be achieved. 


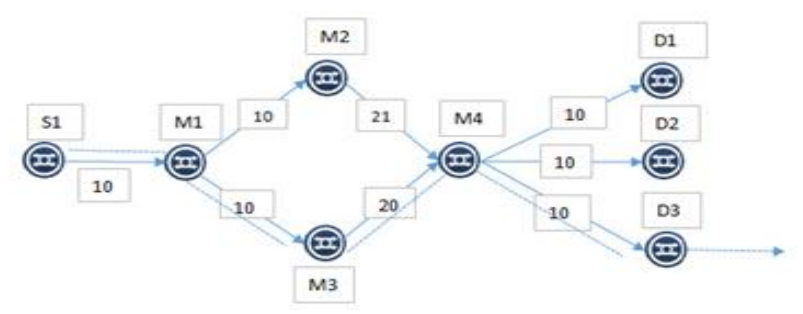

Fig. 2. IGP routing process with modified metric.

\section{B. Traffic Engineering}

Traffic Engineering (TE) applications provides Service Providers a tool to optimized their Internet Protocol routing scheme to enhanced legacy IGP protocols such as IS-IS and OSPF. By mapping IP packets to suitable traffic flows. Transmit traffic flow across MPLS network forwarding engine. Tailor specific network resource to specific network traffic flow [3]. Boarder Gateway Protocol (BGP) [4] as the main Internet Exterior Gateway Protocol (EGP) is responsible for transporting packets across areas and autonomous systems (AS). BGP fell short in the support of Internet traffic engineering, as it becomes a necessity to optimize the Internet backbone. Traffic engineering can be a manual or automated process through a number of network tools and controls [5] such as data resources, control mechanisms, and management tools. Traffic engineering was implemented in IP networks, now it becomes part of the MPLS domain. TE is used to manipulate network traffic to achieve certain objectives. For instance, link utilization can be determined by the ratio of used bandwidth in relation to the allocated bandwidth. This can be achieved by a uniform distribution of network traffic across the network. On the other hand, traffic engineering can also be enacted to optimize limited network resources. One of traffic engineering goals is to distribute available link bandwidth in relation to the required connection, avoiding delays and oversubscribed links. Load balancing will be achieved in both resource allocation and optimal routing choices [6].

TE extensions were implemented in some of IGP routing algorithms such as OSPF-TE and Resource Reservation Protocol (RSVP) [7], [8]. Bandwidth Allocation for Minimizing Resource Usage with Restoration (BAMRUR) introduced in [9] claims to obtain an optimal set of valid paths and traffic distribution by linear programming. BAMRUR objective function is to minimize the bandwidth assigned to a path with certain constraints related to the bandwidth assigned to an active path and the total bandwidth needed. One of the main objectives that was achieved by traffic engineering is cost reduction on Service Providers and that in terms also reduces the cost of business and end users

\section{Multi-protocol Label Switching (MPLS)}

MPLS addresses network routing issues, scalability, and network performance [10]. MPLS works with different networks platforms; such as IP backbone networks, Asynchronous Transfer Mode (ATM) networks, and other technologies. Fig. 3 shows how network traffic is traverse from a sender to a receiver via an MPLS network. MPLS maps an IP address to a fixed length tag known as a label for network packet forwarding. It works with protocols at both the data link and the network layers of the OSI Model. An
MPLS router is called a Label Switch Router (LSR); it inspects the label and the additional fields in forwarding the packet.

At the ingress LSR of an MPLS domain, IP packets are routed based on the information carried in the IP header.

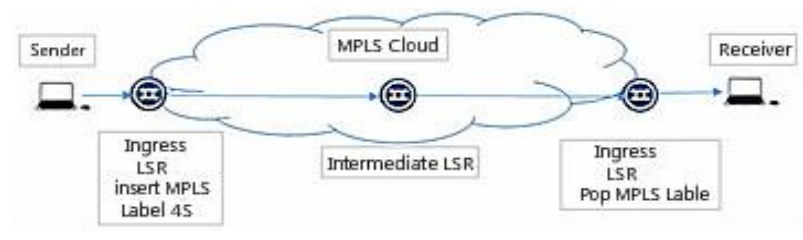

Fig. 3. MPLS network.

\section{Equal Cost Multi-path (ECMP)}

Equal Cost Multi-path is a routing mechanism that allows for the transmission of data packets across multiple path with equal cost. It is up to the forwarding device to determine what the next-hop would be. Couple of well-known routing protocols namely; Open Shortest Path First (OSPF) [11] and Intermediate System to Intermediate System (IS-IS) [12] support Equal-Cost Multipath (ECMP) [13]. ECMP is a routing protocol for transmitting data packets across multi-paths with equal cost from the ingress to the egress node.

Load balancing over multiple paths is one of the mechanisms that Internet Service Providers use to manage their network traffic load. Load balancing has a few benefits: it helps in capacity planning, it reduces traffic delays, and it offers a reliable fault tolerance, since network traffic can pass across other candidate paths [14]. ISPs are striving to provide the optimal available bandwidth for their commercial and business users.

Load balancing requires the use of a key inserted in an available field in the packet. Finding the right field in a packet to use for load balancing is difficult. In the past, the extra encapsulation required fairly deep packet inspection to identify the right field at every hop that the packet traverses. Some of the issues that were noted is the performance issue in relation to the algorithm computational time. Disruption is another concern that manifested with traffic flow changes its path.

\section{RESEARCH WORK}

\section{A. Research Problem}

Literature shows that there are four main problems regarding network bandwidth allocation:

- Inefficient allocation of network bandwidth capacity.

- Underutilized and over utilized network links in relation to network flow traffic request

- Links failure, network congestions, under reliable network connectivity, and network traffic delays resulted in unsatisfied users.

- Costly network design and network operation cost center due to the above problems.

\section{B. Research Objectives}

1) Efficient allocation of network links bandwidth capacity across multiple paths. 
2) Optimum distribution of network traffic flow across multiple paths

3) Choose optimum network paths based on the shortest path first algorithms (SPF). Paths can selected on both joint and disjoint paths. Our research paper will use the disjoint paths options.

4) The overall objective of this research can be summarize in minimize network cost, maximize links capacity utilization, with network traffic engineering concept in mind, optimize user traffic flow.

\section{Research Methodology}

1) Identify multiple network paths for network traffic payload from ingress to egress.

2) Implement the Shortest Path First algorithm (SPF) to choose the paths that traverse the user's network traffic from source node to destination node.

3) Implement the Constrained Shortest Path First Algorithm (CSPF) to select the paths that meet certain requirements.

4) Updates network capacity availability (NCA) every five seconds to provide real time information regarding available links bandwidth capacity.

5) Load sharing of network traffic payload across multiple paths based on the link and path capacity. Network traffic should be allocated proportional across network multiple paths based on links and paths capacity.

\section{Research Model}

The network model is a directed graph with the notations shown in Table I:

TABLE I: NETWORK NOTATIONS

\begin{tabular}{|l|l|}
\hline Notation & Description \\
\hline$N$ & $(N=n)$ is a set of nodes at a given time on the network \\
\hline$L$ & $\begin{array}{l}(L=l) \text { is the set of active network links that capable of } \\
\text { transmitting network traffic between two nodes }\end{array}$ \\
\hline$G(N, L)$ & Directed network graph topology \\
\hline$S D$ & $\begin{array}{l}S D \text { is the set of all source (ingress) and destination } \\
\text { (egress) pairs. }\end{array}$ \\
\hline$c(l)$ & Link's Capacity for link $l$ \\
\hline$q(l)$ & Link $l$ Residual bandwidth \\
\hline$P_{s d}$ & Network routing path from $s$ to $d$ \\
\hline$P_{S D}$ & All set of routing paths from $S$ to $D$ \\
\hline
\end{tabular}

The objective of the Traffic engineering routing algorithms is to route network traffic demand on a reserved bandwidth for each pair of source $(s)$ destination $(d)$ nodes. The bandwidth is reserved for duration of the data transmission from ingress to egress node. Link Available Capacity function (LAC) is shown below.

$$
\mathrm{LAC}=\mathrm{TC}-\mathrm{RC}-\mathrm{BC}
$$

LAC $=$ Link's Available Capacity

$\mathrm{TC}=$ Total Link Capacity

$\mathrm{RC}=$ Link Reserved Capacity

$\mathrm{BC}=$ Link Busy Capacity

One of the two models to this research is, Bandwidth Allocation for Minimizing Resource Usage with Allocation (BAMRUR) introduced in [15].

$$
\sum_{i}^{k}=1\left(C_{\alpha}\right)\left(p_{\kappa}\right)-n_{\kappa}
$$

BAMMUR objective is a min-max bandwidth utilization
Constraint (1) dictates that the sum of bandwidth allocated to the active path is equal to the total bandwidth needed. Constraint (2) validates that the bandwidth given to each active path doesn't exceed the available bandwidth. Constraint (3) requires that, the maximum link usage for all links must be less than the maximum of link utilization.

On the other hand the Proportional Bandwidth Allocation with Path Protection (PBAPP) [15] focuses more in multipath routing and failure recovery of the system. Proportional Bandwidth Allocation with Restoration (PBAR) is different from PBAPP is that PBAR considers all possible paths as useable paths. When a link fails, and prior to connection termination, the PBAR algorithm activates the first feasible path with the highest bandwidth capacity and reroutes the traffic on the new link.

The cost function of the path computation depends on a number of variables. Lee et al. [16] considers the following paths computation cost function: Coststatic $=\int 1$ (bandwidth, hop-count, delay, error ratio).

Step 2 to split the traffic among the candidate paths is as follows: Cost dynamic $=\int 2$ (blockage, packet loss rate, measured delay, jitter)

Given the network model as directed graph $G=(V, E)$, where $V$ is the set of nodes and $E$ is the set of links. The capacity of directional link $(i, j) \subseteq E$ is cij. Each traffic demand $k \subseteq K$ is for a mode pair between an source $s_{k}$ and destination $t_{k}$. The variable $X_{k y}$ denotes the fraction of the traffic demand $k$ assigned to link $(i, j)$. The integer variable $M_{y}^{k}$ denotes how many units of basic discrete split demands for a traffic demand $\mathrm{k}$ are assigned to link $(i, j)$. Let $d k$ be a scaling factor to normalize total traffic demand from the source to become 1. The Mixed Integer Programming problem (MIP) for the bifurcation case is formatted as follows [16]:

Minimize $\alpha$ subject to

$$
\begin{aligned}
& \sum_{f}^{n}:(i, j) \in E^{X_{i j}^{k}}-\sum_{f}^{n}:(i, j) \in E^{X_{j i}^{k}}=0 . k \in K, i \neq s_{k}, t_{k} \\
& \sum_{f}^{n}:(i, j) \in E^{X_{i j}^{k}}-\sum_{f}^{n}:(i, j) \in E^{X_{j i}^{k}}=1 . k \in K, i=s_{k} \\
& \sum_{f}^{n}:(i, j) \in E^{X_{i j}^{k}}-\sum_{f}^{n}:(i, j) \in E^{X_{j i}^{k}}=-1 . k \in K, i=t_{k} \\
& \sum_{f}^{n}:(i, j) \in E^{d_{k} \cdot x_{i j}^{k}} \leq C_{i j}, \alpha(i, J) \in E \\
& X_{i j}^{k}=M_{i j}^{k} \cdot g \\
& 0 \leq X_{i j}^{k} \leq 1 \quad 0 \leq g \leq 1 \quad 00 \leq \leq 1 \quad 0 \leq g \quad 0 \leq \alpha 1 \\
& M_{i j}^{k} \in Z \quad 0 \leq M_{i j}^{k} \leq|1 / g|
\end{aligned}
$$

Constrained Shortest Path First (CSPF), is a subset of the Shortest Path First Algorithm (SPFA). The path generated when using CSPF is basically a shortest path meeting certain constraints. The protocol executes the shortest path algorithm after it excludes the links that don't meet the required constraints. A constraint could be the least bandwidth needed 
per link, maximum number of hop counts between source and destination, minimum class of service required, tolerable end-to-end network congestions.

From our MATLAB Shortest Path Code with No Constraints. In this example the constraints is zero. Fig. 5 shows MATLAB Shortest Path Computation with No Constraints. Path1: from $S>2>8>T$, Path2: from $S>3>9>$ $T$, Path3: from $S>1>4>8>T$, and Path4: $S>2>5>8>T$. As shown additional paths are generated. We noticed some of the paths have equal costs, additional constraints are be added as a tie breaker, or the algorithm may choose either one randomly.

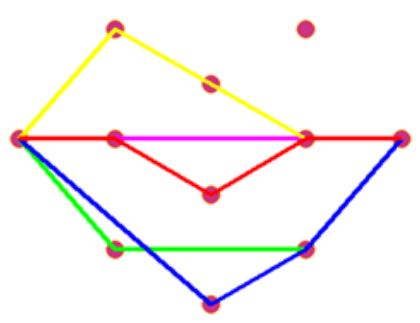

Nodes - Path 1 - Path:2

Fig. 5. MATLAB shortest path computation with no constraint.

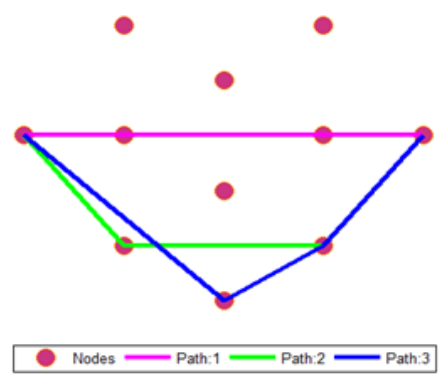

Fig. 6. MATLAB shortest path code with constraints equal 15 units.

Fig. 5 and Fig. 6, show MATLAB shortest path code with capacity constraint, and MATLAB Shortest Path Plot with Capacity Constraint Applied. Only three paths are shown Path1, Path2, and Path3. The shortest path algorithm ran after pruning Path4 and path since they violate the bandwidth capacity constraints.

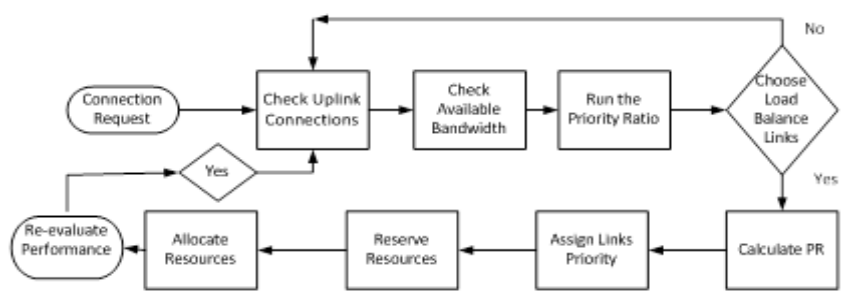

Fig. 7. Network model flowchart.

Fig. 7 shows the network model flowchart that describes the data flow from the connection request. Then an algorithm checks the uplink to make certain that the link is up and operational. Another test is initialed to confirm that the link is operational and passing network traffic. An idle link can be initiated and activated. The available bandwidth calculated according to equation \#1. To confirm that the data payload requirements are met. At the same time the number of paths got determine at this point. The number of paths is determined by the data payload need to be transmitted and the available bandwidth on each path. Priority ratio algorithm is initiated to determine how the data payload is allocated across multiple paths. The dynamic paths can be built at this junction and the resource is allocated accordingly.

\section{CONCLUSIONS}

The overall objective of almost all corporate businesses is to reduce network operational cost, provide positive user experience, and attack additional customers. To achieve these objectives efficient and relatable network resources must be available, for all customers and at all times. The goal of this research is to work towards accomplishing these objectives. In summary, this research uses the k-shortest path to identify the best shortest path from source to destination network. Implement the constrained-base shortest path first (CSPF) to choose those shortest paths which meet certain network traffic requirements. Future work to tackle the second half of the research which is to distribute the network traffic flow across multiple paths proportionally base on each path capacity.

This framework can be implemented in network fault tolerance, load balancing, and MPLS traffic engineering with Entropy Label [14]. In future papers I will describe in details the framework building blocks and give numerical of algorithm implementation.

\section{REFERENCES}

[1] Internet World Stats, Usage, and Population Statistics. [Online] Available: http://www.internetworldstats.com/stats.htm

[2] DARPA Internet Program, Protocol Specification - Internet Protocol, RFC 791, September 1981.

[3] Techtarget. [Online]

http://techtarget.com/definition/traffic-engineering

Available:

[4] Y. Rekhter et al., A Border Gateway Protocol 4, RFC 4271, Jan. 2006

[5] Technical Report. UCAN-CL-TR, University of Cambridge, Computer Laboratory. [Online]. Available: http://www.cl.cam.ac.uk/techreports/UCAM-CL-TR-532.pdf

[6] I. Cidon, R. Rom, and Y. Shavitt, "Multi-path routing combined with resource reservation," in Proc. Sixteenth Annual Joint Conference of the IEEE Computer and Communications Societies, 1997, pp. 92-100.

[7] D. Awduche et al., RSVP-TE: Extensions to RSVP for LSP Tunnels, RFC 3209, December 2001.

[8] D. Katz, K. Kompella, and D. Yeung, Traffic Engineering (TE) Extension to OSPF Version 2, RFC 3630, September 2003.

[9] X. Yu, G. Geng, K. Gay, and C. Siew, "An integrated design of multipath routing with failure survivability in MPLS networks," in Proc. IEEE Computer and Communications Societies, 2004, vol. 1.

[10] A. Viswanathan, E. Rosen, and R. Callon, Multiprotocol Label Switching Architecture, RFC 3031, January 2001.

[11] J. Moy, Open Shortest Path First (OSPF Version 2), RFC 1247, 1981

[12] J. P. Vasseur, N. Shen, and R. Aggarwal, Intermediate System to Intermediate System (IS-IS) Extensions, RFC 4971, July 2007.

[13] C. Hopps, Analysis of an Equal-Cost Multi-Path Algorithm, RFC 2992, November 2000.

[14] K. Kompella et al., The Use of Entropy Labels in MPLS Forwarding, Draft-IETF-MPLS-Entropy-Label-00.

[15] X. Yu, G. Geng, K. Gay, and C. Siew, "An integrated design of multipath routing with failure survivability in MPLS networks," in Proc. IEEE Computer and Communications Societies, 2004, vol. 1.

[16] K. Lee et al., "Hybrid multipath routing algorithms for load balancing in MPLS based IP network," in Proc. the IEEE 20th Int. Conference on Advanced Information Networking and Applications, 2006.

Hatim Hussein is a $\mathrm{PhD}$ student in George Mason University, Fairfax, Virginia, US. He is currently working as an information technology assistant professor at the Northern Virginia Community College, Alexandria, Virginia, USA. He got an undergraduate degree in management science in 1990, from University of Khartoum, Sudan, an MBA degree from Howard University in Washington DC, USA in 1996, and a MS degree in information systems from George Mason University, 2011. His research areas mainly focuses on network traffic engineering. 